\title{
Aberrant white matter microstructure in treatment-resistant schizophrenia
}

\section{Article}

\section{Accepted Version}

Creative Commons: Attribution-Noncommercial-No Derivative Works 4.0

McNabb, C. B. ORCID: https://orcid.org/0000-0002-6434-5177, Mcllwain, M. E., Anderson, V. M., Kydd, R. R., Sundram, F. and Russell, B. R. (2020) Aberrant white matter microstructure in treatment-resistant schizophrenia. Psychiatry Research: Neuroimaging, 305. 111198. ISSN 0925-4927 doi: https://doi.org/10.1016/j.pscychresns.2020.111198 Available at https://centaur.reading.ac.uk/93222/

It is advisable to refer to the publisher's version if you intend to cite from the work. See Guidance on citing.

To link to this article DOI: http://dx.doi.org/10.1016/j.pscychresns.2020.111198

Publisher: Elsevier

All outputs in CentAUR are protected by Intellectual Property Rights law, including copyright law. Copyright and IPR is retained by the creators or other copyright holders. Terms and conditions for use of this material are defined in the End User Agreement.

\section{www.reading.ac.uk/centaur}

\section{CentAUR}


Central Archive at the University of Reading

Reading's research outputs online 
Aberrant white matter microstructure in treatment-resistant schizophrenia

${ }^{+}$Carolyn B. McNabb ${ }^{a, b},{ }^{+}$Meghan E. Mcllwain ${ }^{a}$, Valerie M. Anderson ${ }^{a}$, Robert R. Kydd ${ }^{c}$, Frederick Sundram ${ }^{c}$ * Bruce R. Russell ${ }^{d}$

aSchool of Pharmacy, University of Auckland, 85 Park Road, Grafton, Auckland 1023, New Zealand

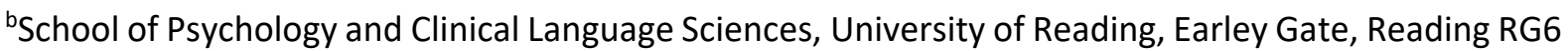
7BE, United Kingdom

'Department of Psychological Medicine, University of Auckland, Auckland City Hospital, 2 Park Road, Grafton, Auckland 1023, New Zealand

${ }^{d}$ School of Pharmacy, University of Otago, PO Box 56, Dunedin 9054, New Zealand

+Joint first authors

Corresponding author:

Dr Bruce Russell

Tel +64 34797272

Fax +64 34797034

Email: bruce.russell@otago.ac.nz

School of Pharmacy, University of Otago

PO Box 56, Dunedin, 9054, New Zealand 


\section{Abstract}

Treatment response in schizophrenia divides into three subcategories: treatment-responsive (firstline responders; FLR), treatment-resistant (TRS), and ultra-treatment-resistant schizophrenia (UTRS). White matter abnormalities could drive antipsychotic resistance but little work has investigated differences between TRS and UTRS. The current study aimed to establish whether differences in white matter structure are present across both treatment-resistant subtypes or if UTRS is distinct from TRS. Diffusion-weighted images were acquired for 18 individuals with TRS, 14 with UTRS, 18 FLR and 20 healthy controls. Measures of fractional anisotropy (FA), mean diffusivity (MD), radial diffusivity (RD) and axial diffusivity ( $A D)$ were obtained using tract-based spatial statistics. Analysis of variance and post-hoc t-tests were conducted for each measure. Those with TRS had lower FA than healthy controls in superior longitudinal fasciculus, corpus callosum, thalamic radiation, corticospinal tract, internal capsule, corona radiata and fronto-occipital fasciculus ( $p<.05 \mathrm{FWE}$-corrected). Lower FA was also observed in TRS compared with UTRS in the superior longitudinal fasciculus ( $p<.05$ FWEcorrected). No post-hoc tests survived corrections for multiple comparisons and no differences in $M D, A D$ or RD were observed. These data suggest that microstructural deficits in white matter could contribute to TRS but suggest that other mechanisms may be more relevant for UTRS.

Keywords:

Fractional anisotropy, ultra-treatment-resistant schizophrenia, treatment response, tract-based spatial statistics 


\section{Introduction}

Pharmacological intervention is the most effective treatment for schizophrenia (Matheson et al., 2014). However, the rate of response to initial treatment with typical or atypical non-clozapine antipsychotics reaches only $60 \%$ to $80 \%$, with response rates to a second non-clozapine antipsychotic as low as 16\% (Agid et al., 2011; Elkis and Buckley, 2016). Resistance to first-line antipsychotic therapy accounts for some of the highest rates of hospitalisation and impaired functioning in mental health (lasevoli et al., 2016; Lieberman and Murray, 2012) and is estimated to require USD 34 billion per annum in direct healthcare costs in the US alone (Kennedy et al., 2014). Furthermore, a recent meta-analysis (Siskind et al., 2017) revealed that clozapine, the most effective treatment for first-line therapy-resistant schizophrenia, is effective in only $40 \%$ of treatmentrefractory individuals, suggesting that $12 \%$ to $20 \%$ of people with schizophrenia are ultra-treatmentresistant. Reflecting on these observations, researchers have posited that these three response levels represent three distinct subtypes of schizophrenia: treatment-responsive (first-line responders; FLR), treatment-resistant (clozapine responders; TRS) and ultra-treatment-resistant schizophrenia (clozapine-resistant; UTRS) (Farooq et al., 2013; Lee et al., 2015).

A growing body of research investigating potential differences in structural brain organisation between response subtypes of schizophrenia lends support to the concept of treatment-based categorical distinctions in this disorder and suggests that differences in white matter structure are present even at first episode of psychosis (Chen et al., 2018; Reis Marques et al., 2014). Reis Marques et al. (2014) reported that, at baseline, individuals with first-episode psychosis who failed to respond to a 12-week course of first-line antipsychotic therapy had lower fractional anisotropy (FA) than either healthy comparison subjects or responders in the left uncinate fasciculus, cingulum and superior longitudinal fasciculus and commissural tracts such as the corpus callosum (responders were indistinguishable from healthy comparison subjects). In support of these findings, Chen et al. (2018) identified white matter impairments in the right temporal and occipital lobes in resistant compared with responsive individuals with first-episode psychosis following one year of antipsychotic treatment. Differences in FA between responders and non-responders to first-line therapy also appear to persist throughout the course of disease. Widespread reductions in FA have been reported in patients who met criteria for TRS prior to commencing clozapine when compared to healthy subjects (Holleran et al., 2013), as well as in those with TRS with severe symptoms compared with non-treatment resistant schizophrenia (Ochi et al., 2020). Researchers also identified lower FA in the body of the corpus callosum in those with clozapine-eligible schizophrenia compared with FLR (McNabb et al., 2018a), as well as reduced mean generalised FA within 12 white matter 
tracts in people with non-remitting compared with remitting schizophrenia (Huang et al., 2018). Two earlier cross-sectional studies of patients with chronic schizophrenia also reported that a poor response to antipsychotic treatment was associated with lower FA in the whole brain and in specific tracts such as the uncinate and superior longitudinal fasciculi (Luck et al., 2011; Mitelman et al., 2006).

Although these studies provide mounting evidence for a role of white matter aberrations in treatment-resistant subtypes of schizophrenia, they have focused primarily on comparisons with healthy controls and differences between FLR and those with TRS (or poor treatment outcome), either omitting those with UTRS or absorbing them into the TRS subtype. If a diagnosis of schizophrenia really does divide into three distinct response subtypes, studies should be designed to distinguish between FLR, individuals with TRS and those with UTRS. Therefore, the current study sought to investigate white matter markers of treatment response in people with schizophrenia using the classification scheme proposed by Farooq et al. (2013) and Lee et al. (2015); specifically categorising the disorder into FLR, TRS and UTRS subtypes.

\section{Methods}

\subsection{Participants}

Details of participant recruitment have been described previously (Anderson et al., 2015; Goldstein et al., 2015; McNabb et al., 2018b). Individuals with a diagnosis of schizophrenia according to the Diagnostic and Statistical Manual of Mental Disorders (DSM-IV) were recruited from mental health services (a community mental health centre or forensic psychiatric inpatient unit) in Auckland, New Zealand. Participants were enrolled into one of three study arms (FLR, TRS or UTRS). Those who were responding well to first-line atypical antipsychotic monotherapy were assigned to the FLR group; response was defined as an improvement of positive symptoms according to standard practice and current treatment guidelines for schizophrenia (Lehman et al., 2004; McGorry, 2005). Those who had failed at least two previous six-to-eight-week trials of atypical antipsychotics and were receiving clozapine at the time of screening were assigned to the TRS group and participants who had failed at least two previous six-to-eight-week trials of atypical antipsychotics and had also failed an adequate trial of clozapine monotherapy (at least 8 weeks post titration (Mouaffak et al., 2006)) were assigned to the UTRS group. Participants in TRS and UTRS groups met minimum requirements for diagnosis of TRS or UTRS, respectively, according to Treatment Response and Resistance in Psychosis (TRRIP) working group guidelines (Howes et al., 2016). All participants' 
clinical records were inspected by a registered pharmacist (MM) to ensure they had received adequate trials of antipsychotics at appropriate dosages for an appropriate length of time and to ensure that those in the UTRS cohort had been switched from clozapine monotherapy due to poor treatment response rather than poor adherence or clozapine-induced side effects. Patients in all treatment groups were required to be at most mildly ill, determined using the Clinical Global Impression (CGI) (which corresponds to a score of 3 out of 7 using this scale; 7 being the most extremely ill) to control for state effects (as otherwise differences in the TRS/UTRS groups could be ascribed to the presence of more severe symptoms). A healthy comparison group with no history of mental or neurological illness was recruited by advertising in the community. Age, sex and ethnicity were matched on a group basis. All participants were between the ages of 18 and 45 years. Exclusion criteria were as follows: history of traumatic brain injury (loss of consciousness for more than three minutes), neurological illness, significant physiological comorbidity or contraindication to magnetic resonance imaging (MRI). The study was approved by the Northern X Regional Ethics Committee (reference: NTX/09/05/042) and conducted in accordance with the Helsinki Declaration as revised in 1989. All participants gave informed written consent - ability to give informed consent was judged by the treating psychiatrist.

Duration of psychosis and Positive and Negative Syndrome Scale (PANSS) scores (Kay et al., 1987) were assessed at study entry by a study investigator (MM) who had received professional PANSS training (including use of consensus videos) by a registered psychiatrist. Antipsychotic dose at the time of assessment was converted to chlorpromazine equivalents (CPZE) using formulae with power transformation (Andreasen et al., 2010). In the absence of a power formula for amisulpride, CPZE were calculated using expert consensus regarding antipsychotic dosing (Gardner et al., 2010). Past and present substance abuse was evaluated using the Alcohol, Smoking and Substance Involvement Screening Test (ASSIST; World Health Organisation) at study entry. Participants also provided a urine sample, which was screened for the presence of amphetamine, methamphetamine, benzodiazepines, cocaine, opiates and tetrahydrocannabinol (THC) (Medix Pro-Split Integrated Cup, Multi Drug Screening Test; Sobercheck Ltd). Standardised premorbid intelligence quotient (IQ) scores were determined using a computerised version of the "spot the real word" task within the IntegNeuro test battery (Brain Resource Company, Sydney, Australia) (Baddeley et al., 1993; Gordon, 2003; Gordon et al., 2005). The sample size was based on previous studies examining the relationship between treatment outcomes and diffusion tensor imaging (DTI) measures in schizophrenia (Garver et al., 2008; Luck et al., 2011; Mitelman et al., 2009) and compares with more recent studies investigating white matter microstructure in individuals with treatment-resistant 
schizophrenia (e.g. 20 FLR + 20 TRS (Chen et al., 2018), 27 Controls +28 FLR + 24 TRS (Ochi et al., 2020)).

\subsection{Image acquisition and pre-processing}

Scanning was performed using a 3T Siemens Magnetom Skyra (Siemens, Germany). A 32-channel head coil was used for the majority of scans; where the participant could not fit comfortably into this coil, a 20-channel head coil was used $(F L R=2, \operatorname{TRS}=1$, UTRS $=2$; see Supplementary Materials for further details). T1-weighted images were acquired using a magnetization-prepared 180-degrees radio-frequency pulses and rapid gradient-echo (MPRAGE) sequence (Brant-Zawadzki et al., 1992). Acquisition parameters were as follows: repetition time (TR) $1900 \mathrm{~ms}$; echo time (TE) $2.39 \mathrm{~ms}$; inversion time (TI) 900 ms; flip angle $9^{\circ}$; repetition 1; acceleration factor 2; field of view (FOV) 230 $\mathrm{mm}$; matrix $256 \times 256$; voxel size $0.9 \times 0.9 \times 0.8 \mathrm{~mm}$. Diffusion-weighted images were acquired using an echo planar imaging (EPI) sequence with the following parameters: phase encoding $A>>P, T R$ $8900 \mathrm{~ms}$, TE $95 \mathrm{~ms}$, FOV $240 \mathrm{~mm}$, matrix 122 x 122, $2.0 \mathrm{~mm}$ slice thickness, isotropic voxel size 2.0 x $2.0 \times 2.0 \mathrm{~mm}$. An acceleration factor (GRAPPA) of 2 was used. Sixty-seven slices were acquired in the anterior to posterior direction with diffusion-weighting factor $b=1000 \mathrm{~s} / \mathrm{mm}^{2}$ in 64 unique gradientencoding directions. Eight scans without diffusion weighting $(b=0)$ were also acquired. All scans were visually examined for artefacts including subject motion, excess signal-to-noise and incomplete acquisitions. No participants were excluded on this basis.

Image preprocessing and analysis were performed using the FMRIB Software Library (FSL) (Jenkinson et al., 2012). Raw diffusion-weighted images were corrected for head motion and eddy current distortions using FSL's eddy tool (Andersson and Sotiropoulos, 2016). In-scanner head motion from the first volume and the previous volume were determined and averaged across volumes to give two root mean squared values for each participant. Susceptibility-induced distortions were corrected using FSL's fugue function and output registered to gradient-free images using the linear registration function (FLIRT) (Jenkinson et al., 2002).

Measures of white matter microstructure, including FA, mean diffusivity (MD), axial diffusivity (AD) and radial diffusivity (RD) were obtained using Tract-Based Spatial Statistics (TBSS) (Smith et al., 2006), part of FSL (Smith et al., 2004). FA images were created by fitting a tensor model to the raw diffusion data using FMRIBs Diffusion Toolbox (FDT), and then brain-extracted using the Brain Extraction Tool (BET) (Smith, 2002). All subjects' FA data were then aligned to the FMRIB58_FA target image using the nonlinear registration tool FNIRT (Andersson et al., 2007) which uses a bspline representation of the registration warp field (Rueckert et al., 1999) and transformed into 
Montreal Neurologic Institute (MNI)-152 standard space. Next, a mean FA skeleton representing the centres of all white matter tracts common to the group was created and thinned at a threshold of 0.3 to exclude low anisotropic regions. Each subject's aligned FA data were then projected onto this skeleton. Anatomical localisation of significant white matter clusters was determined using the Juelich histological white matter labels and tractography atlases. The same approach was used for preprocessing of $M D, A D$ and $R D$ data.

\subsection{Statistical analysis}

Whole-brain statistical analyses were performed using FSL's Randomise tool. Threshold-free cluster enhancement was used for statistical comparisons, utilising a nonparametric permutation test in which group membership was permuted 5,000 times to generate a null distribution for each contrast. An analysis of variance (ANOVA) was employed to investigate whether any difference between groups existed in the data. Post hoc unpaired $t$-tests were then used to explore potential differences between the groups. Age and sex were demeaned prior to analysis and included as covariates of no interest in both ANOVA and post hoc analyses (Bose et al., 2009). Additional regression correlations (Pearson) were performed to investigate the associations between white matter measures and participant head motion, as well as daily antipsychotic dose in CPZE. All voxelwise $p$-values were corrected for multiple comparisons using a Family-Wise Error (FWE) correction threshold of $p=.05$; post hoc tests were corrected for multiple comparisons using the False Discovery Rate (FDR). Both corrected and uncorrected post hoc $t$-test results are reported.

Head motion during scanning can introduce bias that may influence microstructural measures of white matter (Ling et al., 2012). To evaluate the effect of motion on FA, we conducted a regression analysis between FA and root mean squared motion from first and previous volumes.

Analyses of socio-demographic and clinical characteristics were conducted using the Psych and DescTools packages for R (R Core Development Team, 2012; Revelle, 2017; Signorell et al., 2019). ANOVA was used to compare continuous variables with Bonferroni correction for multiple comparisons of post hoc tests. Fisher's Exact test was used for comparisons of non-parametric data. FA images were created in FSLeyes and plots were created using the ggplot2 package in R (Wickham, 2016).

\section{Results}

\subsection{Participants}


A total of 70 participants ( 20 healthy controls, 18 FLR, 18 individuals with TRS and 14 with UTRS) were included in the final analysis. Demographic and clinical data are summarized in Table 1. There were no significant differences in gender, age, IQ or positive tests for THC between the groups, though ASSIST scores were higher in FLR and those with UTRS compared with healthy controls. No differences in duration of illness, duration of untreated psychosis, PANSS total scores, or any of the subscales were observed between the groups with schizophrenia. Those with UTRS were receiving a higher dose of antipsychotics (measured in CPZE) at the time of scanning compared with both FLR and those with TRS; clusters demonstrating differences in FA between treatment groups were therefore correlated against CPZE to determine whether antipsychotic dose affected the results. The same was done for ASSIST scores and cluster FA.

Root mean squared motion from the first volume and the previous volume were evaluated for each participant. No statistically significant differences in motion were identified between treatment groups (see Table 1).

\subsection{TBSS results}

An ANOVA between all study groups revealed a statistically significant effect for FA $(p=.048)$, indicating an effect of group on white matter microstructure. No such effect was observed for MD, $A D$ or RD. Pairwise comparisons between groups (FWE-corrected) revealed lower FA in those with TRS compared to healthy controls (figure $1 ; p=.022$ uncorrected) as well as those with UTRS (figure $2 ; p=.030$ uncorrected). Neither test survived corrections for multiple post hoc comparisons between groups using the FDR; however, the consistency of our findings with the literature to date suggests that this is most likely a result of small sample size as opposed to false positive results.

Clusters with lower FA in those with TRS are shown in figure 3 and described further in table 2. Those with TRS had lower FA than controls in the superior longitudinal fasciculus (bilateral), corpus callosum, thalamic radiation (bilateral), corticospinal tract (bilateral), internal capsule (left), corona radiata (bilateral) and fronto-occipital fasciculus (bilateral), among other regions. In contrast, differences between those with TRS and UTRS were confined to the right superior longitudinal fasciculus (including the temporal component of the superior longitudinal fasciculus).

To further interrogate the differences between groups, mean FA for each cluster was determined for every participant (figure 4). FA was lowest in those with TRS for all clusters and, for the most part, was similar for FLR and those with UTRS, both of which had slightly lower FA than healthy controls. The exception was for the single cluster exhibiting lower FA in those with TRS compared with UTRS, 
in which the effect appears to be driven by a combination of low FA in those with TRS and high FA in those with UTRS.

\subsection{Correlation with clinical characteristics}

As CPZE demonstrated differences between treatment groups, we ran additional regression analyses investigating the relationships between CPZE and white matter measures. There was no area identified where FA, MD, AD or RD correlated with antipsychotic daily dose in CPZEs. Likewise, no statistically significant relationship between cluster FA and antipsychotic dose measured in CPZE was identified when measured independently for each cluster (figure 5). Similar analyses were conducted for PANSS total score and age at onset of psychosis in those with schizophrenia and no significant relationships to cluster FA values were identified (see supplementary figures S1 and S2, respectively).

\subsection{Correlation with socio-demographic characteristics}

As previous work found significant associations between IQ and FA (Luders et al., 2007; Luders et al., 2011; Navas-Sánchez et al., 2014), for each cluster, we ran correlations between mean FA and standardised (premorbid) IQ. IQ correlated with mean FA in two clusters (figure 6), although only one survived corrections for multiple comparisons across clusters (Bonferroni-corrected $p$ threshold .007). The significant correlation was for a cluster spanning only 5 voxels (located in the left external capsule / inferior fronto-occipital fasciculus / uncinate fasciculus) whereby those with TRS had lower FA than healthy controls. The remaining clusters showed no relationship between FA and IQ.

Recreational drug-use (measured using the ASSIST) was higher in FLR and those with UTRS compared with controls. In addition, as lifetime drug use, in particular use of THC (Epstein and Kumra, 2015), may influence white matter microstructure in tracts relevant to schizophrenia, we ran Pearson's correlations between ASSIST scores and FA values in each cluster. One participant failed to complete the ASSIST and was excluded from the analysis. Results are presented in figure S3. No statistically significant relationship between ASSIST score and cluster FA was identified for any cluster.

\subsection{Effect of head motion on FA}

Root mean squared motion did not significantly correlate with FA in the regression analysis. However, adding motion values as regressors of no interest in the main ANOVA resulted in a loss of significant group effect.

\section{Discussion}


Results from studies investigating white matter microstructure in people with schizophrenia have contributed toward a consensus that people who fail to respond to first-line antipsychotic therapy exhibit greater disruptions in FA than treatment responders (Chen et al., 2018; Huang et al., 2018; McNabb et al., 2018a; Ochi et al., 2020; Reis Marques et al., 2014). However, schizophrenia encompasses two distinct subtypes of treatment resistance - clozapine responsive treatment resistance (TRS) and clozapine resistant treatment resistance (UTRS). This was the first study to explicitly define and compare these two subtypes in addition to FLR and healthy controls to investigate possible white matter markers of treatment resistance in people with schizophrenia. Importantly, treatment resistance in the current study was evaluated on the basis of past treatment failure in addition to current treatment response (i.e. did the person respond to treatment with clozapine monotherapy?) - Therefore, TRS and UTRS groups should be interpreted as clozapine monotherapy-responsive and clozapine monotherapy-resistant, respectively, rather than exhibiting increasing degrees of symptom severity.

Our results suggest that TRS and UTRS are likely distinct from one another in their white matter profiles, TRS exhibiting lower FA than both healthy controls and UTRS. Therefore, resistance to the clinical benefits of first-line antipsychotic drugs and subsequent sensitivity to clozapine may be attributable to global disruptions in FA. By comparison, those with UTRS had FA comparable to FLR and healthy controls, suggesting that white matter disruptions play a less pivotal role in treatment resistance for these individuals. A caveat of the current research is that head motion (although not significantly different between groups, see Table 1) was higher in healthy controls than those with TRS. Adding head motion as a regressor in the analysis led to a non-significant effect of group. With limited sample size, this loss of significant effect may be attributable to a loss in degrees of freedom, but could reflect real differences in motion between groups contributing to the overall group effect on FA in the main analysis. We therefore caution that these results be interpreted with care, though their resemblance to previous findings (Chen et al., 2018; Huang et al., 2018; Luck et al., 2011; Mitelman et al., 2006; Ochi et al., 2020; Reis Marques et al., 2014) suggests that they do make a valuable contribution to the literature.

Prominent regions of white matter disruptions in our TRS cohort included the superior longitudinal fasciculus (including the temporal region of this tract), body and splenium of the corpus callosum and forceps major, corticospinal tract, superior and posterior corona radiata, posterior limb of the internal capsule, anterior and posterior thalamic radiation, the external capsule, uncinate fasciculus, inferior fronto-occipital fasciculus and cerebral peduncle. Our findings of widespread white matter aberrations in those with TRS are in agreement with previous studies investigating white matter 
microstructure in responders and non-responders to first-line therapy. Luck et al. (2011) identified reduced FA in people with chronic schizophrenia experiencing poor response compared with healthy controls within the superior longitudinal fasciculus and uncinate fasciculus but not the cingulum, with FA reductions more pronounced in the poor outcome compared with good outcome group (Luck et al., 2011). Likewise, Reis Marques et al. (2014) found that those who went on to develop a poor response to antipsychotic treatment exhibited decreased FA across multiple white matter regions, including the left uncinate, cingulum and superior longitudinal fasciculus and corpus callosum, compared to healthy controls. The corpus callosum is the fundamental white matter structure conferring interhemispheric connectivity. Lower FA in the forceps major, splenium and body of the corpus callosum in our TRS group is in agreement with previous studies investigating treatment resistance, as well as schizophrenia more generally. Decreased FA in the corpus callosum is consistently reported in patients with schizophrenia (Bora et al., 2011; Foong et al., 2000; Kong et al., 2011; Kubicki et al., 2008; Patel et al., 2011) and more specifically chronic schizophrenia (Douaud et al., 2007; Koch et al., 2010; Kubicki et al., 2008; Mitelman et al., 2009; Miyata et al., 2010; Rotarska-Jagiela et al., 2008). Mitelman et al. observed lower average FA in the corpus callosum of individuals with schizophrenia with poor-outcome compared with good outcome (Mitelman et al., 2009). Lower FA in the body of the corpus callosum has also been observed in people with schizophrenia who are eligible for clozapine compared with those responding well to first-line antipsychotic therapy (McNabb et al., 2018a). Though we did not detect differences between FLR and TRS patient groups in the current study, FA was lowest in the group with TRS throughout the corpus callosum body, splenium and forceps major, suggesting that callosal aberrations are most severe in this population.

Our finding of lower FA in the fronto-occipital fasciculus may be driven by disruptions in myelination in those with TRS. A recent study investigating magnetic resonance markers of myelination found lower myelin water fraction in people with schizophrenia compared with healthy controls in this region, although no difference between resistant and responsive patients were observed (Vanes et al., 2018). Myelin water fraction within the corpus callosum was also associated with cognitive control in those with schizophrenia. In their study, Vanes et al. (2018) explicitly excluded individuals receiving clozapine to avoid the introduction of subgroups including ultra-treatment-resistant schizophrenia. However, we have demonstrated that this subgroup exhibits white matter patterns quite distinct from TRS, suggesting that distinguishing TRS from UTRS provides useful information about the treatment response patterns of individuals with schizophrenia. 
Although we did not demonstrate significant differences in white matter between all schizophrenia cohorts and healthy controls in the current analysis, a previous analysis of the same participants identified lower white matter volume in all schizophrenia groups compared with controls (Anderson et al., 2015). Therefore, the lower (but not statistically significant) FA observed in FLR (and to a lesser extent those with UTRS) compared with healthy controls could indicate a less severe but still clinically relevant reduction in FA in these individuals.

Differences in FA between controls and TRS as well as between those with TRS and UTRS appear to be strongly lateralised in the current study. However, increasing the statistical threshold to include "trend-level" ( $p \leq .1)$ differences in FA revealed a more bilateral pattern of FA deficits in those with TRS (data not shown). As statistically significant clusters identified in the current analysis had $p$ values close to the threshold value of $p=.05$, the lateralisation observed here may be a feature of the statistical cut-off rather than indicating true lateralised disruption in white matter microstructure. Further studies in larger samples will be necessary to determine whether any biologically meaningful conclusions can be made from this supposed lateralisation.

All participants in the TRS group and the majority of participants in the UTRS groups were receiving clozapine at the time of scanning. Previous studies have associated both a reduction in white matter volume (Molina et al., 2005) and increase in FA (Ozcelik-Eroglu et al., 2014) with the use of clozapine. As our results demonstrate lower FA in those on clozapine monotherapy and similar levels of FA in those with UTRS (receiving augmented clozapine therapy) and FLR, it is unlikely that our effects are driven by exposure to clozapine. However, if FA is lowest in those with TRS and clozapine increases FA, this could be a mechanism by which clozapine improves symptoms of the disorder.

Current antipsychotic dose is also unlikely to have driven the effects observed in this study. As the UTRS group were receiving significantly higher doses of antipsychotics (measured in CPZE) compared with both FLR and those with TRS, we ran correlations within the whole brain and significant FA clusters to determine the effects of CPZE. No significant effects were observed in either analysis, suggesting that the lower FA in those with TRS is independent of antipsychotic exposure. Although no effect of current dosage was found, as it was not possible to obtain cumulative lifetime exposure to antipsychotics (due to limited information available in public health records), we cannot rule out the potential for more long-term effects of antipsychotic dosage to have impacted FA. Likewise, family history of psychiatric symptoms was not accounted for in the current study but may have had some influence on our findings. 
Although past and present recreational drug use was higher in FLR and those with UTRS compared with healthy controls, no relationship between ASSIST scores and FA were identified for any cluster. These results suggest that the lower FA identified in those with TRS compared with controls and those with UTRS is unlikely to be driven by the effects of recreational drug use on white matter microstructure.

We did, however, identify a relationship between IQ and mean FA in one of the seven clusters (located in the external capsule) demonstrating differences between TRS and healthy controls. IQ has been shown to correlate with white matter microstructure in the external capsule in previous research (Navas-Sánchez et al., 2014); however, as the cluster demonstrating a significant effect contained only 5 voxels, it is unlikely that IQ is driving the white matter differences between those with TRS and healthy controls. In addition, the remaining clusters showed no such relationship, therefore we suggest that the relationship between IQ, treatment resistance and microstructural measures of white matter is weak at best.

This study benefits from a well characterised cohort of participants, well matched for clinical and demographic variables including gender, age, duration of illness, age at onset, duration of untreated psychosis and premorbid IQ. Participants taking clozapine had well-established TRS and patient groups were stabilized on their current treatment. Inclusion of patients with mild symptoms provides us with confidence that the differences in FA observed in those with TRS are unlikely to be the result of more severe active symptoms in this cohort. Correlation analysis with total PANSS scores supports this theory.

Participants in the current study were responding well to treatment, with PANSS scores (mean total score 59.5-61.2) corresponding to mild symptom severity (Leucht et al., 2005). The decision to recruit well-responding participants was based on the desire to minimise interference from current symptoms on white matter measures but also to ensure that those in the FLR and TRS groups were truly responsive to first-line and clozapine monotherapy, respectively.

This study has several limitations. Recent work has shown that the use of different head coils (namely 8- and 32-channel coils) can introduce positive bias when analysing white matter microstructure (Panman et al., 2019). However, the bias introduced by the use of 20- and 32-channel coils (as in the current study) is likely to be far less than that introduced by the use of 8- and 32channel coils. Although the use of both 20- and 32-channel head coils in the current study is a limitation, our decision not to exclude these participants based on head size was carefully considered. Recruitment of individuals with schizophrenia who meet the strict criteria set out in this 
study is challenging and we believe the benefit of including more participants outweighs the potential bias introduced by the small number of participants with larger head size. In our study, individuals scanned with the 20-channel coil were well distributed among the treatment groups (two participants in the FLR group, one in the TRS group and two in the UTRS group) as well as in terms of their FA values (triangles in figures 4-6, S1 and S2). It is therefore unlikely that the use of this data introduced FA bias. Including the head coil as a regressor of no interest in the analysis resulted in a non-statistically significant effect of group on FA ( $p=.0548)$. As inclusion of the coil regressor resulted in only a small increase in $p$-value, it is unlikely to have contributed substantially to the effects observed in the main analysis. This is nonetheless an important consideration for future work.

The sample size was comparable to other studies investigating white matter microstructure in treatment-resistant schizophrenia (Chen et al., 2018; Luck et al., 2011; Mitelman et al., 2006; Ochi et al., 2020), though would be considered relatively small compared with other neuroimaging studies. Studies involving larger cohorts of FLR, TRS and UTRS participants are required to confirm the results of our and other studies in this area (though (Reis Marques et al., 2014) found similar results of decreased FA in those with TRS in a study of 52 controls, 30 FLR and 33 people with TRS).

The study's cross-sectional design imparts a degree of uncertainty about the underlying cause of observed differences. However, these findings are in line with previous work highlighting differences between controls and non-responders to antipsychotic therapy, suggesting the effects are robust. Therefore, although our post hoc analyses did not survive corrections for multiple comparisons between groups, their concordance with previous work provides a strong foundation on which a theory attributing treatment resistance to white matter abnormalities can be built.

In summary, these results suggest that microstructural white matter abnormalities in people with schizophrenia are more pronounced in those with TRS than those who respond well to first-line antipsychotics and those with UTRS and that these aberrations exist despite effective treatment with clozapine. Future work is required to confirm the differences in FA values identified between those with TRS and UTRS in the current study and to evaluate whether differences between TRS and UTRS are present before initiation of clozapine. We hope these findings, in combination with the current literature, will encourage researchers to delineate between treatment-resistant and ultra-treatment resistance schizophrenia in the future.

Acknowledgements 
This work was supported by The University of Auckland Faculty Research and Development Fund, with support from the New Zealand Schizophrenia Research Group and the Oakley Mental Health Research Foundation. Meghan Mcllwain was supported by a University of Auckland Doctoral Scholarship and a New Zealand Federation of Graduate Women Fellowship. Valerie Anderson was supported by an Edith C. Coan Research Fellowship from The Auckland Medical Research Foundation.

The authors would like to thank the Waitakere and Counties Manukau District Health Boards for their cooperation during the study as well as the participants.

No funders had any role in the study design; in the collection, analysis or interpretation of data; in the writing of the report; or in the decision to submit the article for publication.

\section{Contributions}

Carolyn McNabb conducted the final analysis and wrote the final version of this manuscript, Meghan Mcllwain assisted with ethics approval, recruited participants, confirmed medication histories, conducted and scored PANSS interviews, oversaw imaging, contributed to data analysis and wrote the first draft of the manuscript. Valerie Anderson assisted with imaging and contributed to data analysis. Robert Kydd provided expertise about the study population, confirmed patient diagnosis, and assisted with PANSS scoring. Frederick Sundram also confirmed diagnoses, and contributed to data analysis. Bruce Russell was the PI and obtained funding, ethics approval, provided expertise about the study population, treatment regimens and interpretation of the findings. All authors contributed to and have approved the final manuscript.

\section{References}

Agid, O., Arenovich, T., Sajeev, G., Zipursky, R.B., Kapur, S., Foussias, G., Remington, G., 2011. An algorithm-based approach to first-episode schizophrenia: response rates over 3 prospective antipsychotic trials with a retrospective data analysis. J. Clin. Psychiatry 72, 1439-1444. Anderson, V.M., Goldstein, M.E., Kydd, R.R., Russell, B.R., 2015. Extensive Gray Matter Volume Reduction in Treatment-Resistant Schizophrenia. Int. J. Neuropsychopharmacol. 18, pyv016. Andersson, J.L., Jenkinson, M., Smith, S., 2007. Non-linear registration, aka Spatial normalisation FMRIB technical report TR07JA2 from www.fmrib.ox.ac.uk/analysis/techrep Andersson, J.L.R., Sotiropoulos, S.N., 2016. An integrated approach to correction for off-resonance effects and subject movement in diffusion MR imaging. Neuroimage 125, 1063-1078. 
Andreasen, N.C., Pressler, M., Nopoulos, P., Miller, D., Ho, B.-C., 2010. Antipsychotic dose equivalents and dose-years: a standardized method for comparing exposure to different drugs. Biol. Psychiatry 67, 255-262.

Baddeley, A., Emslie, H., Nimmo-Smith, I., 1993. The Spot-the-Word test: a robust estimate of verbal intelligence based on lexical decision. Br. J. Clin. Psychol. 32 ( Pt 1), 55-65.

Bora, E., Fornito, A., Radua, J., Walterfang, M., Seal, M., Wood, S.J., Yucel, M., Velakoulis, D., Pantelis, C., 2011. Neuroanatomical abnormalities in schizophrenia: a multimodal voxelwise metaanalysis and meta-regression analysis. Schizophr. Res. 127, 46-57.

Bose, S.K., Mackinnon, T., Mehta, M.A., Turkheimer, F.E., Howes, O.D., Selvaraj, S., Kempton, M.J., Grasby, P.M., 2009. The effect of ageing on grey and white matter reductions in schizophrenia. Schizophr. Res. 112, 7-13.

Brant-Zawadzki, M., Gillan, G.D., Nitz, W.R., 1992. MP RAGE: a three-dimensional, T1-weighted, gradient-echo sequence--initial experience in the brain. Radiology 182, 769-775.

Chen, M., Zhuo, C.-J., Qian, L.-J., Wang, B., Zhai, J.-G., Ji, F., Ke, X.-Y., 2018. Specific White Matter Impairments in Patients with Treatment-Refractory First-Episode Schizophrenia: A 1-Year Follow-up Pilot Study. Chin. Med. J. 131, 879-880.

Douaud, G., Smith, S., Jenkinson, M., Behrens, T., Johansen-Berg, H., Vickers, J., James, S., Voets, N., Watkins, K., Matthews, P.M., James, A., 2007. Anatomically related grey and white matter abnormalities in adolescent-onset schizophrenia. Brain 130, 2375-2386.

Elkis, H., Buckley, P.F., 2016. Treatment-resistant schizophrenia. Psychiatr. Clin. North Am. 39, 239265.

Epstein, K.A., Kumra, S., 2015. White matter fractional anisotropy over two time points in early onset schizophrenia and adolescent cannabis use disorder: A naturalistic diffusion tensor imaging study. Psychiatry Research: Neuroimaging 232, 34-41.

Farooq, S., Agid, O., Foussias, G., Remington, G., 2013. Using treatment response to subtype schizophrenia: Proposal for a new paradigm in classification. Schizophr. Bull. 39, 1169-1172. Foong, J., Maier, M., Clark, C.A., Barker, G.J., Miller, D.H., Ron, M.A., 2000. Neuropathological abnormalities of the corpus callosum in schizophrenia: a diffusion tensor imaging study. J. Neurol. Neurosurg. Psychiatry 68, 242-244.

Gardner, D.M., Murphy, A.L., O'Donnell, H., Centorrino, F., Baldessarini, R.J., 2010. International consensus study of antipsychotic dosing. Am. J. Psychiatry 167, 686-693.

Garver, D.L., Holcomb, J.A., Christensen, J.D., 2008. Compromised myelin integrity during psychosis with repair during remission in drug-responding schizophrenia. Int. J. Neuropsychopharmacol. 11, 49-61.

Goldstein, M.E., Anderson, V.M., Pillai, A., Kydd, R.R., Russell, B.R., 2015. Glutamatergic Neurometabolites in Clozapine-Responsive and-Resistant Schizophrenia. Int. J.

Neuropsychopharmacol. 18, pyu117.

Gordon, E., 2003. Integrative neuroscience. Neuropsychopharmacology 28 Suppl 1, S2-8.

Gordon, E., Cooper, N., Rennie, C., Hermens, D., Williams, L.M., 2005. Integrative neuroscience: the role of a standardized database. Clin. EEG Neurosci. 36, 64-75.

Holleran, L., Ahmed, M., Schmidt, H., McFarland, J., Emsell, L., Leemans, A., Scanlon, C., Dockery, P., McCarthy, P., Barker, G.J., McDonald, C., Cannon, D.M., 2013. Altered Interhemispheric and Temporal Lobe White Matter Microstructural Organisation in Severe Chronic Schizophrenia. Neuropsychopharmacology.

Howes, O.D., McCutcheon, R., Agid, O., Bartolomeis, A.d., van Beveren, N.J., Birnbaum, M.L., Bloomfield, M.A., Bressan, R.A., Buchanan, R.W., Carpenter, W.T., Castle, D.J., Citrome, L., Daskalakis, Z.J., Davidson, M., Drake, R.J., Dursun, S., Ebdrup, B.H., Elkis, H., Falkai, P., Fleischacker, W.W., Gadelha, A., Gaughran, F., Glenthøj, B.Y., Graff-Guerrero, A., Hallak, J.E.C., Honer, W.G., Kennedy, J., Kinon, B.J., Lawrie, S.M., Lee, J., Leweke, F.M., MacCabe, J.H., McNabb, C.B., Meltzer, H., Möller, H.-J., Nakajima, S., Pantelis, C., Marques, T.R., Remington, G., Rossell, S.L., Russell, B.R., Siu, C.O., Suzuki, T., Sommer, I.E., Taylor, D., Thomas, N., Üçok, A., Umbricht, D., Walters, J.T.R., Kane, J., 
Correll, C.U., 2016. Treatment-resistant schizophrenia: Treatment response and resistance in psychosis (TRRIP) working group consensus guidelines on diagnosis and terminology. Am. J. Psychiatry, appi. ajp. 2016.16050503.

Huang, J.-Y., Liu, C.-M., Hwang, T.-J., Chen, Y.-J., Hsu, Y.-C., Hwu, H.-G., Lin, Y.-T., Hsieh, M.-H., Liu, C.C., Chien, Y.-L., Tseng, W.-Y.I., 2018. Shared and distinct alterations of white matter tracts in remitted and nonremitted patients with schizophrenia. Hum. Brain Mapp. 39, 2007-2019. lasevoli, F., Giordano, S., Balletta, R., Latte, G., Formato, M.V., Prinzivalli, E., De Berardis, D., Tomasetti, C., de Bartolomeis, A., 2016. Treatment resistant schizophrenia is associated with the worst community functioning among severely-ill highly-disabling psychiatric conditions and is the most relevant predictor of poorer achievements in functional milestones. Prog.

Neuropsychopharmacol. Biol. Psychiatry 65, 34-48.

Jenkinson, M., Bannister, P., Brady, M., Smith, S., 2002. Improved optimization for the robust and accurate linear registration and motion correction of brain images. Neuroimage 17, 825-841.

Jenkinson, M., Beckmann, C.F., Behrens, T.E., Woolrich, M.W., Smith, S.M., 2012. FSL. Neuroimage 62, 782-790.

Kay, S.R., Fiszbein, A., Opler, L.A., 1987. The positive and negative syndrome scale (PANSS) for schizophrenia. Schizophr. Bull. 13, 261-276.

Kennedy, J.L., Altar, C.A., Taylor, D.L., Degtiar, I., Hornberger, J.C., 2014. The social and economic burden of treatment-resistant schizophrenia: a systematic literature review. Int. Clin.

Psychopharmacol. 29, 63-76.

Koch, K., Wagner, G., Dahnke, R., Schachtzabel, C., Schultz, C., Roebel, M., Gullmar, D., Reichenbach, J.R., Sauer, H., Schlosser, R.G., 2010. Disrupted white matter integrity of corticopontine-cerebellar circuitry in schizophrenia. Eur. Arch. Psychiatry Clin. Neurosci. 260, 419-426.

Kong, X., Ouyang, X., Tao, H., Liu, H., Li, L., Zhao, J., Xue, Z., Wang, F., Jiang, S., Shan, B., Liu, Z., 2011. Complementary diffusion tensor imaging study of the corpus callosum in patients with first-episode and chronic schizophrenia. J. Psychiatry Neurosci. 36, 120-125.

Kubicki, M., Styner, M., Bouix, S., Gerig, G., Markant, D., Smith, K., Kikinis, R., McCarley, R.W., Shenton, M.E., 2008. Reduced interhemispheric connectivity in schizophrenia-tractography based segmentation of the corpus callosum. Schizophr. Res. 106, 125-131.

Lee, J., Takeuchi, H., Fervaha, G., Sin, G.L., Foussias, G., Agid, O., Farooq, S., Remington, G., 2015. Subtyping schizophrenia by treatment response: antipsychotic development and the central role of positive symptoms. Can. J. Psychiatry. 60, 515-522.

Lehman, A.F., Lieberman, J.A., Dixon, L.B., McGlashan, T.H., Miller, A.L., Perkins, D.O., Kreyenbuhl, J., McIntyre, J.S., Charles, S.C., Altshuler, K., 2004. Practice guideline for the treatment of patients with schizophrenia. Am. J. Psychiatry 161.

Leucht, S., Kane, J.M., Kissling, W., Hamann, J., Etschel, E., Engel, R.R., 2005. What does the PANSS mean? Schizophr. Res. 79, 231-238.

Lieberman, J.A., Murray, R.M., 2012. Comprehensive care of schizophrenia: A textbook of clinical management. Oxford University Press.

Luck, D., Buchy, L., Czechowska, Y., Bodnar, M., Pike, G.B., Campbell, J.S., Achim, A., Malla, A., Joober, R., Lepage, M., 2011. Fronto-temporal disconnectivity and clinical short-term outcome in first episode psychosis: a DTI-tractography study. J. Psychiatr. Res. 45, 369-377.

Luders, E., Narr, K.L., Bilder, R.M., Thompson, P.M., Szeszko, P.R., Hamilton, L., Toga, A.W., 2007. Positive correlations between corpus callosum thickness and intelligence. Neuroimage 37, 14571464.

Luders, E., Thompson, P.M., Narr, K.L., Zamanyan, A., Chou, Y.-Y., Gutman, B., Dinov, I.D., Toga, A.W., 2011. The link between callosal thickness and intelligence in healthy children and adolescents. Neuroimage 54, 1823-1830.

Matheson, S., Shepherd, A., Carr, V., 2014. How much do we know about schizophrenia and how well do we know it? Evidence from the Schizophrenia Library. Psychol. Med. 44, 3387-3405. 
McGorry, P., 2005. Royal Australian and New Zealand College of Psychiatrists clinical practice guidelines for the treatment of schizophrenia and related disorders. Aust. N. Z. J. Psychiatry 39, 1-30. McNabb, C.B., Kydd, R., Sundram, F., Soosay, I., Russell, B.R., 2018a. Differences in white matter connectivity between treatment-resistant and treatment-responsive subtypes of schizophrenia. Psychiatry Research: Neuroimaging 282, 47-54.

McNabb, C.B., Tait, R.J., Mcllwain, M.E., Anderson, V.M., Suckling, J., Kydd, R.R., Russell, B.R., 2018b. Functional network dysconnectivity as a biomarker of treatment resistance in schizophrenia. Schizophr Research 195, 160-167.

Mitelman, S.A., Newmark, R.E., Torosjan, Y., Chu, K.-W., Brickman, A.M., Haznedar, M.M., Hazlett, E.A., Tang, C.Y., Shihabuddin, L., Buchsbaum, M.S., 2006. White matter fractional anisotropy and outcome in schizophrenia. Schizophr. Res. 87, 138-159.

Mitelman, S.A., Nikiforova, Y.K., Canfield, E.L., Hazlett, E.A., Brickman, A.M., Shihabuddin, L., Buchsbaum, M.S., 2009. A longitudinal study of the corpus callosum in chronic schizophrenia. Schizophr. Res. 114, 144-153.

Miyata, J., Yamada, M., Namiki, C., Hirao, K., Saze, T., Fujiwara, H., Shimizu, M., Kawada, R., Fukuyama, H., Sawamoto, N., Hayashi, T., Murai, T., 2010. Reduced white matter integrity as a neural correlate of social cognition deficits in schizophrenia. Schizophr. Res. 119, 232-239.

Molina, V., Reig, S., Sanz, J., Palomo, T., Benito, C., Sanchez, J., Sarramea, F., Pascau, J., Desco, M., 2005. Increase in gray matter and decrease in white matter volumes in the cortex during treatment with atypical neuroleptics in schizophrenia. Schizophr. Res. 80, 61-71.

Mouaffak, F., Tranulis, C., Gourevitch, R., Poirier, M.-F., Douki, S., Olié, J.-P., Lôo, H., Gourion, D., 2006. Augmentation strategies of clozapine with antipsychotics in the treatment of ultraresistant schizophrenia. Clin. Neuropharmacol. 29, 28-33.

Navas-Sánchez, F.J., Alemán-Gómez, Y., Sánchez-Gonzalez, J., Guzmán-De-Villoria, J.A., Franco, C., Robles, O., Arango, C., Desco, M., 2014. White matter microstructure correlates of mathematical giftedness and intelligence quotient. Hum. Brain Mapp. 35, 2619-2631.

Ochi, R., Noda, Y., Tsuchimoto, S., Tarumi, R., Honda, S., Matsushita, K., Tsugawa, S., Plitman, E., Masuda, F., Ogyu, K., Wada, M., Miyazaki, T., Fujii, S., Chakravarty, M.M., Graff-Guerrero, A., Uchida, H., Mimura, M., Nakajima, S., 2020. White matter microstructural organizations in patients with severe treatment-resistant schizophrenia: A diffusion tensor imaging study. Prog.

Neuropsychopharmacol. Biol. Psychiatry 100, 109871.

Ozcelik-Eroglu, E., Ertugrul, A., Oguz, K.K., Has, A.C., Karahan, S., Yazici, M.K., 2014. Effect of clozapine on white matter integrity in patients with schizophrenia: A diffusion tensor imaging study. Psychiatry Research: Neuroimaging 223, 226-235.

Panman, J.L., To, Y.Y., van der Ende, E.L., Poos, J.M., Jiskoot, L.C., Meeter, L.H.H., Dopper, E.G.P., Bouts, M.J.R.J., van Osch, M.J.P., Rombouts, S.A.R.B., van Swieten, J.C., van der Grond, J., Papma, J.M., Hafkemeijer, A., 2019. Bias Introduced by Multiple Head Coils in MRI Research: An 8 Channel and 32 Channel Coil Comparison. Front. Neurosci. 13.

Patel, S., Mahon, K., Wellington, R., Zhang, J., Chaplin, W., Szeszko, P.R., 2011. A meta-analysis of diffusion tensor imaging studies of the corpus callosum in schizophrenia. Schizophr. Res. 129, 149155.

R Core Development Team, 2012. R: A Language and Environment for Statistical Computing, 1.1.4 ed. R Foundation for Statistical Computing, Vienna.

Reis Marques, T., Taylor, H., Chaddock, C., Dell'Acqua, F., Handley, R., Reinders, A.A.T.S., Mondelli, V., Bonaccorso, S., DiForti, M., Simmons, A., David, A.S., Murray, R.M., Pariante, C.M., Kapur, S., Dazzan, P., 2014. White matter integrity as a predictor of response to treatment in first episode psychosis. Brain 137, 172-182.

Revelle, W.R., 2017. psych: Procedures for personality and psychological research.

Rotarska-Jagiela, A., Schonmeyer, R., Oertel, V., Haenschel, C., Vogeley, K., Linden, D.E., 2008. The corpus callosum in schizophrenia-volume and connectivity changes affect specific regions.

Neuroimage 39, 1522-1532. 
Rueckert, D., Sonoda, L.I., Hayes, C., Hill, D.L.G., Leach, M.O., Hawkes, D.J., 1999. Nonrigid registration using free-form deformations: application to breast MR images. Medical Imaging, IEEE Transactions on 18, 712-721.

Signorell, A., Aho, K., Alfons, A., Anderegg, N., Aragon, T., Arppe, A., Baddeley, A., Barton, K., Bolker, B., Borchers, H.W., Caeiro, F., Champely, S., Chessel, D., Chhay, L., Cummins, C., Dewey, M., Doran, H.C., Dray, S., al., e., 2019. DescTools: Tools for Descriptive Statistics. , 0.99.29 ed.

Siskind, D., Siskind, V., Kisely, S., 2017. Clozapine response rates among people with treatmentresistant schizophrenia: data from a systematic review and meta-analysis. SAGE Publications Sage CA: Los Angeles, CA.

Smith, S.M., 2002. Fast robust automated brain extraction. Human Brain Mapping 17, 143-155. Smith, S.M., Jenkinson, M., Johansen-Berg, H., Rueckert, D., Nichols, T.E., Mackay, C.E., Watkins, K.E., Ciccarelli, O., Cader, M.Z., Matthews, P.M., Behrens, T.E.J., 2006. Tract-based spatial statistics: Voxelwise analysis of multi-subject diffusion data. Neuroimage 31, 1487-1505.

Smith, S.M., Jenkinson, M., Woolrich, M.W., Beckmann, C.F., Behrens, T.E.J., Johansen-Berg, H., Bannister, P.R., De Luca, M., Drobnjak, I., Flitney, D.E., Niazy, R.K., Saunders, J., Vickers, J., Zhang, Y., De Stefano, N., Brady, J.M., Matthews, P.M., 2004. Advances in functional and structural MR image analysis and implementation as FSL. Neuroimage 23, Supplement 1, S208-S219.

Vanes, L.D., Mouchlianitis, E., Wood, T.C., Shergill, S.S., 2018. White matter changes in treatment refractory schizophrenia: Does cognitive control and myelination matter? Neurolmage. Clinical 18, 186-191.

Wickham, H., 2016. ggplot2: elegant graphics for data analysis. Springer.

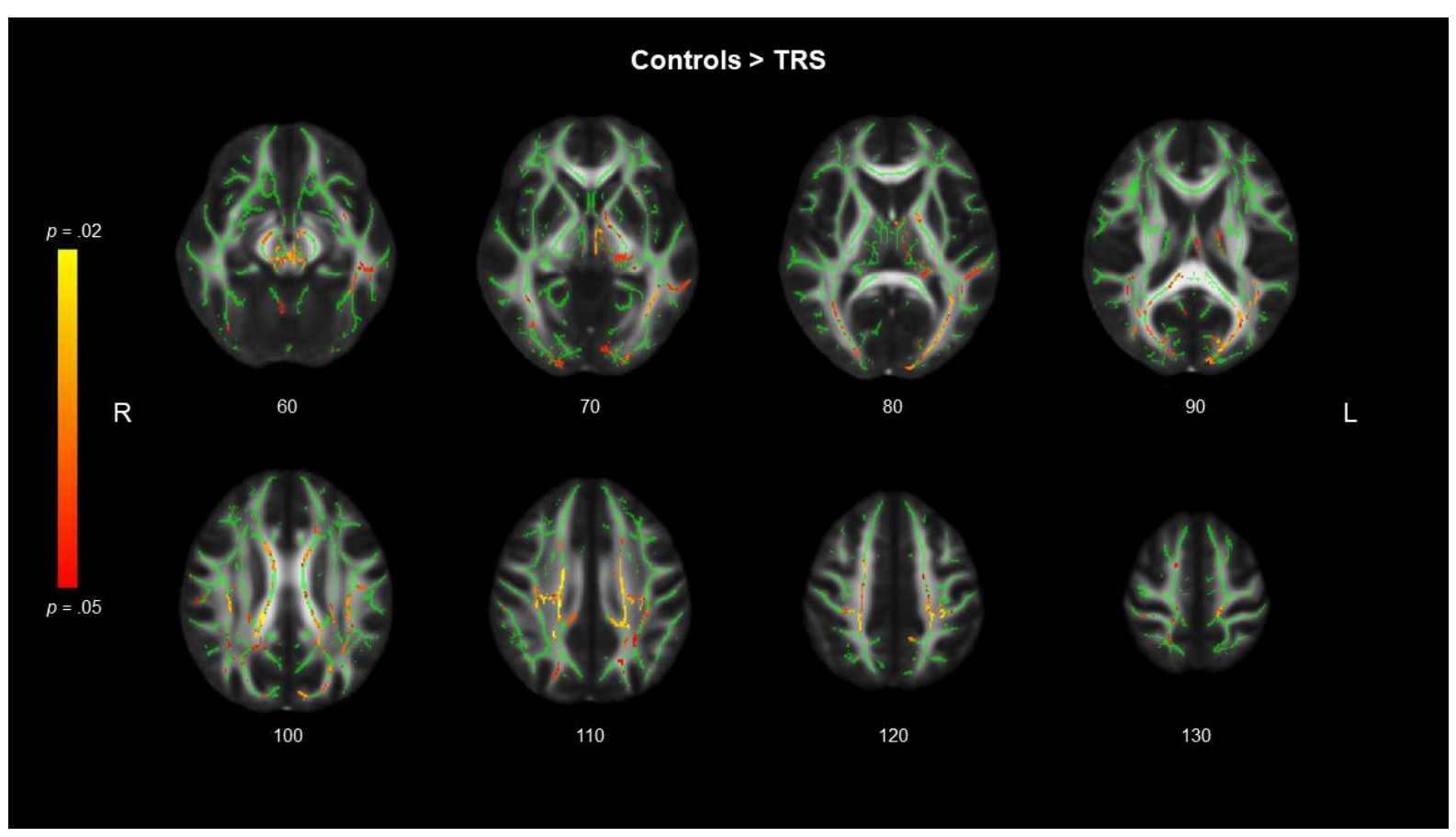

Figure 1. White matter maps showing lower fractional anisotropy (FA) in the treatment resistant schizophrenia (TRS) group compared to psychiatrically healthy controls ( $p<.05$, FWE-corrected). Red-yellow voxels represent regions in which FA is significantly lower in the TRS group relative to healthy controls, overlaid on to the mean FA skeleton (green) and FMRIB58-FA image in standard 
$\mathrm{MNI}-152$ space (radiologic view with Z coordinates). $\mathrm{FWE}=$ family-wise error; $\mathrm{MNI}=$ Montreal Neurological Institute.

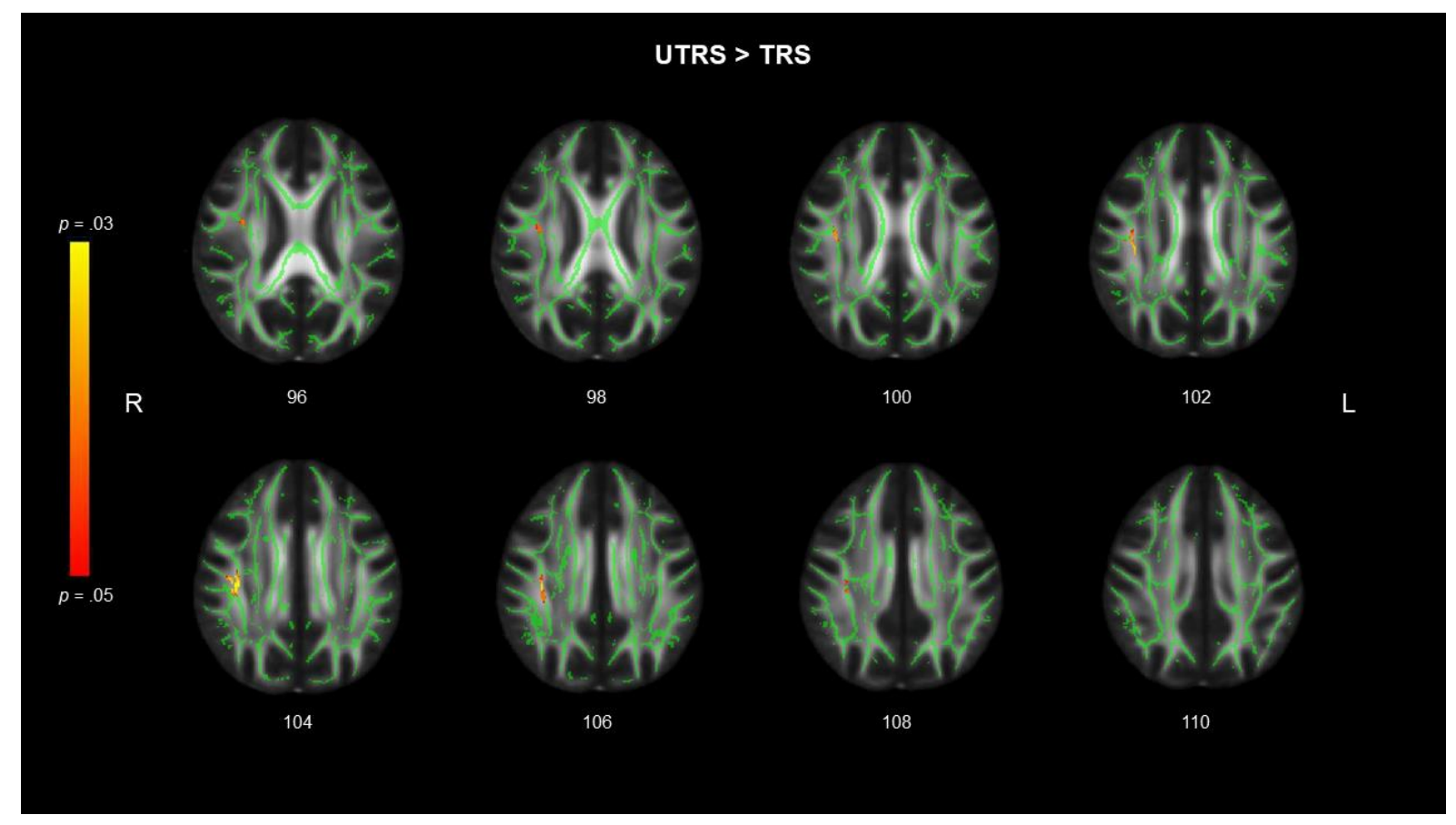

Figure 2. White matter maps showing lower fractional anisotropy $(F A)$ in the treatment-resistant schizophrenia (TRS) group compared to the ultra-treatment-resistant schizophrenia (UTRS) group ( $p$ $<.05$, FWE-corrected). Red-yellow voxels represent regions in which FA is significantly lower in the TRS group relative to UTRS, overlaid on to the mean FA skeleton (green) and FMRIB58-FA image in standard $\mathrm{MNI}-152$ space (radiologic view with Z coordinates). $\mathrm{FWE}=$ family-wise error; $\mathrm{MNI}=$ Montreal Neurological Institute. 


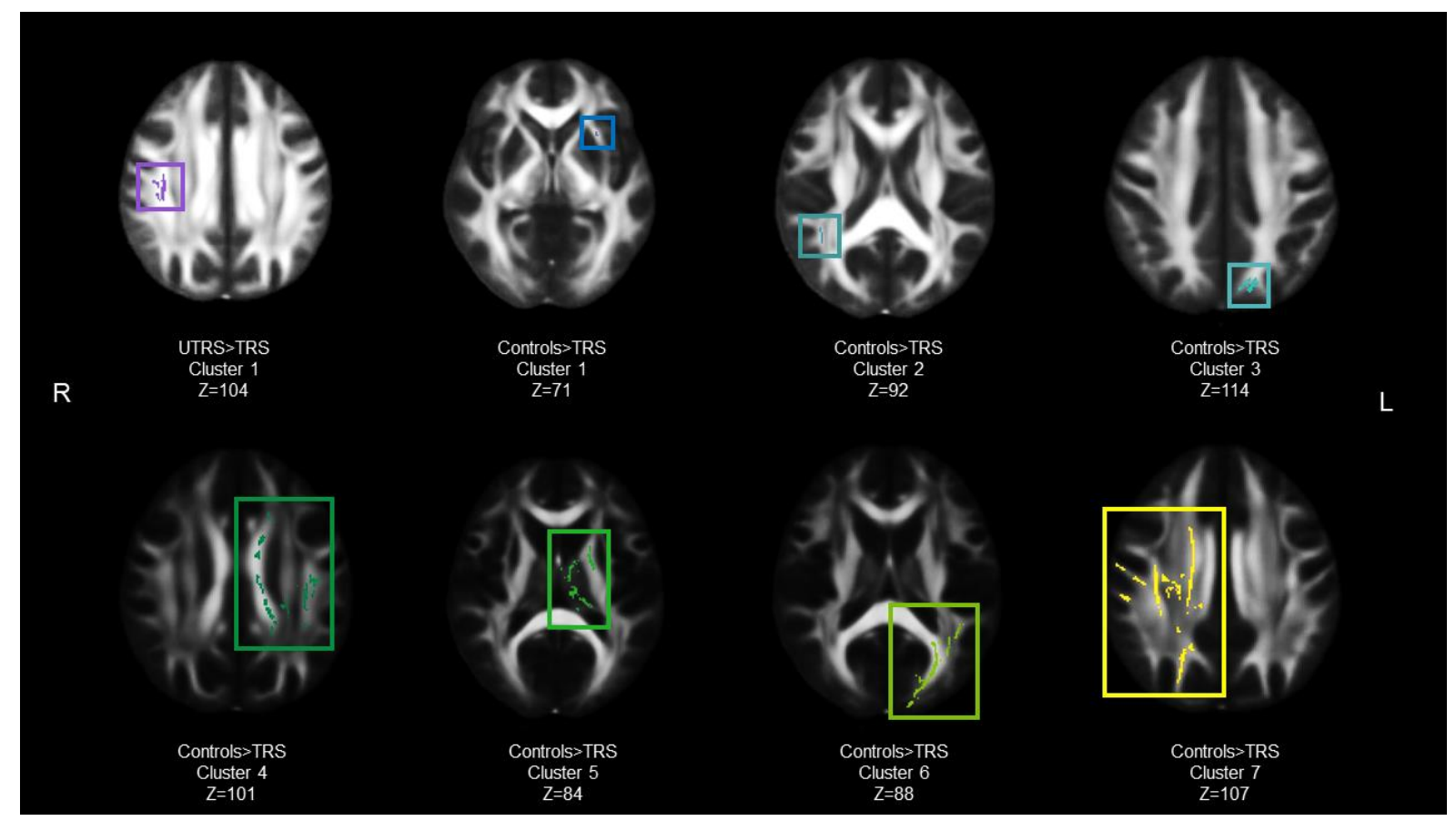

Figure 3. Voxel clusters in which individuals with treatment-resistant schizophrenia (TRS) exhibited lower fractional anisotropy (FA) than psychiatrically healthy controls or those with ultra-treatmentresistant schizophrenia (UTRS).

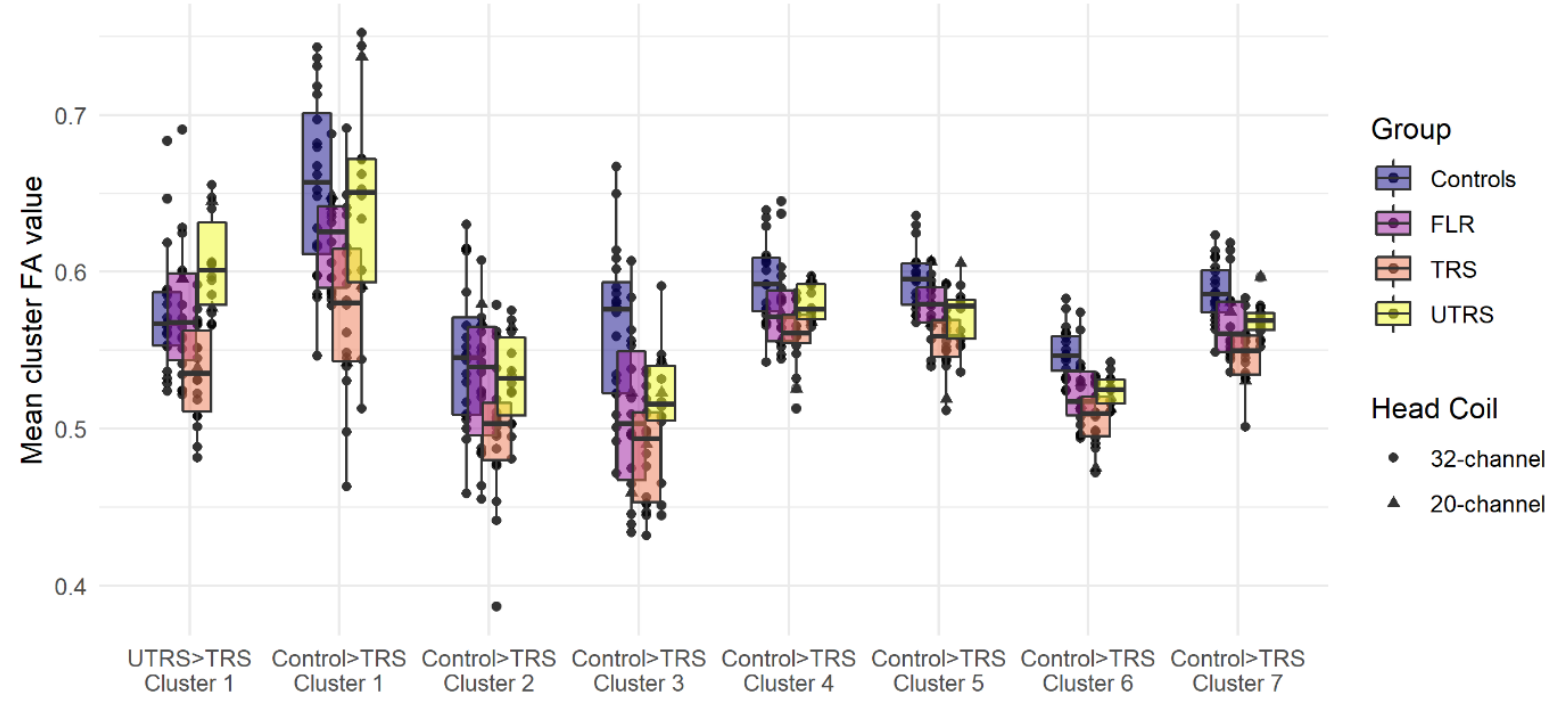

Figure 4. Mean fractional anisotropy (FA) for clusters in which the group with treatment resistant schizophrenia (TRS) had significantly lower FA than healthy comparison subjects or those with ultratreatment-resistant schizophrenia (UTRS). Individual subjects' mean FA values are represented by points, with group data represented by each box - lines through each box represent the group 
median; the upper and lower "hinges" correspond to the first and third quartiles (the 25th and 75th percentiles). Data beyond the end of the whiskers are outliers (as specified by Tukey).

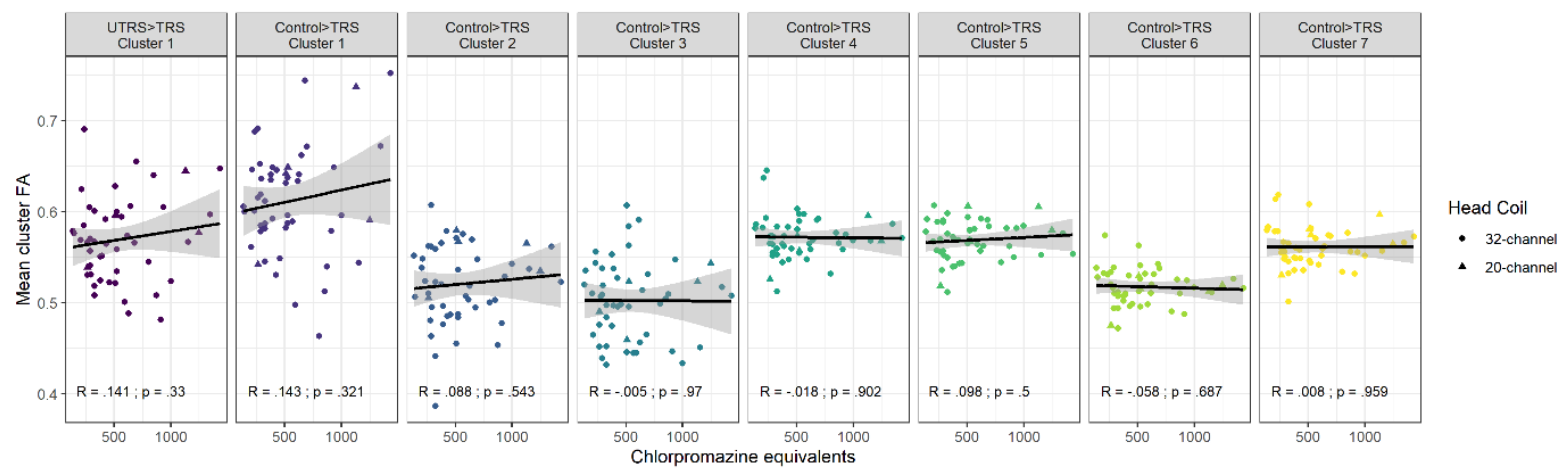

Figure 5. Relationship between mean cluster fractional anisotropy (FA) and antipsychotic dose measured in chlorpromazine equivalents (CPZE) in those with schizophrenia. Pearson's correlation (R) and uncorrected $p$ values are presented for each cluster.

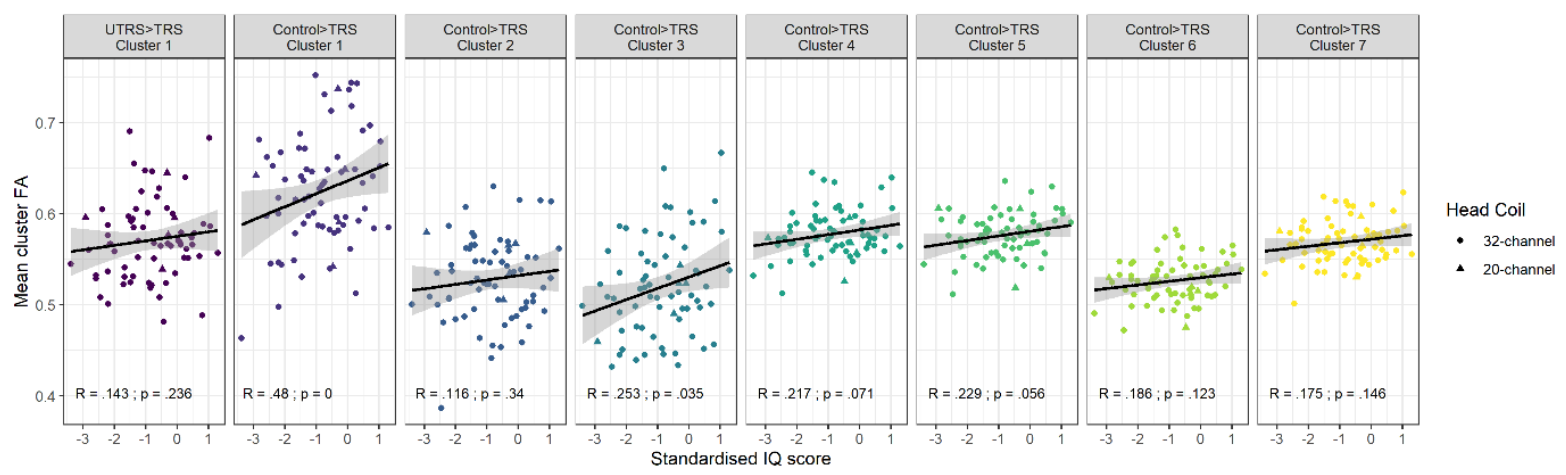

Figure 6. Relationship between mean cluster fractional anisotropy (FA) and standardised (premorbid) IQ. Pearson's correlation (R) and uncorrected $p$ values are presented for each cluster. 
Table 1. Demographic data (mean (SD) unless otherwise stated) of study participants by treatment group. *One participant in the TRS group did not complete the ASSIST questionnaire. ASSIST data are presented for all other participants.

\begin{tabular}{|c|c|c|c|c|c|}
\hline & $\begin{array}{l}\text { Controls } \\
(n=20)\end{array}$ & $\begin{array}{l}\text { FLR } \\
(n=18)\end{array}$ & $\begin{array}{l}\text { TRS } \\
(n=18)\end{array}$ & $\begin{array}{l}\text { UTRS } \\
(n=14)\end{array}$ & Test Statistic \\
\hline Sex (male/female) & $17 / 3$ & $14 / 4$ & $14 / 4$ & $11 / 3$ & $\begin{array}{l}\text { Fisher's Exact test (FET), } \\
p=.933\end{array}$ \\
\hline Age at scan (years) & $33.2(8.4)$ & $32.2(7.9)$ & $33.2(8.2)$ & $34.6(7.0)$ & $F(3,66)=.31, p=.818$ \\
\hline Premorbid IQ (standardized scores) & $-0.838(1.286)$ & $-0.781(0.982)$ & $-0.955(1.170)$ & $-0.885(0.883)$ & $F(3,66)=.08, p=.971$ \\
\hline Age at onset (years) & - & $22.3(5.5)$ & $20.7(4.5)$ & $22.8(6.4)$ & $F(2,47)=.64, p=.531$ \\
\hline Duration of illness (years) & - & $10.0(7.9)$ & $12.5(6.7)$ & $12.2(4.8)$ & $F(2,47)=.73, p=.485$ \\
\hline Duration of untreated psychosis (months) & - & $12.5(14.7)$ & $11.1(18.1)$ & $22.5(27.5)$ & $F(2,47)=1.46, p=.242$ \\
\hline PANSS total score & - & $59.5(11.0)$ & $59.6(14.1)$ & $61.2(10.9)$ & $F(2,47)=.09, p=.911$ \\
\hline PANSS positive subscale score & - & $13.4(5.36)$ & $11.9(5.5)$ & $12.6(5.1)$ & $F(2,47)=.36, p=.703$ \\
\hline PANSS negative subscale score & - & $17.1(5.75)$ & $18.8(6.8)$ & $19.5(7.6)$ & $F(2,47)=.57, p=.572$ \\
\hline PANSS general subscale score & - & $28.9(6.01)$ & $28.8(6.2)$ & $29.1(4.4)$ & $F(2,47)=.01, p=.993$ \\
\hline Current prescribed antipsychotic & - & $\begin{array}{l}\text { Olanzapine }(n=8) \\
\text { Risperidone }(n=6) \\
\text { Aripiprazole }(n=3) \\
\text { Amisulpride }(n=1)\end{array}$ & Clozapine $(n=18)$ & $\begin{array}{l}\text { Clozapine + amisulpride }(n=4) \\
\text { Clozapine + aripiprazole }(n=4) \\
\text { Clozapine + risperidone }(n=2) \\
\text { Clozapine + quetiapine }(n=1) \\
\text { Aripiprazole + quetiapine }(n=2) \\
\text { Risperidone + quetiapine }(n=1)\end{array}$ & \\
\hline
\end{tabular}


Dose at time of scan (chlorpromazine

equivalents)

Positive test for THC (n)

ASSIST total score*
$421.5(191.6)$

0

$26.4(17.3)$

$50.2(21.4)$
$F(2,47)=12.03, p<.001$ UTRS Vs FLR, $p<.001$ UTRS vs TRS, $p<.001$

$F E T, p=.096$

$F(3,65)=5.18, p=.003$

FLR vs Control, $p=.013$

UTRS vs Control, $p=.007$

Root mean squared motion (mm: median,

IQR)

\begin{tabular}{|c|c|c|c|c|c|}
\hline From first volume & $1.03(1.87)$ & $1.07(0.41)$ & $0.83(0.66)$ & $0.94(1.06)$ & $\begin{array}{l}\text { Kruskal-Wallis }(K W) \text { chi } \\
\text { squared test }(3)=2.94, \\
p=.401\end{array}$ \\
\hline From previous volume & $0.23(0.24)$ & $0.32(0.22)$ & $0.29(0.25)$ & $0.25(0.20)$ & $K W(3)=.170, p=.982$ \\
\hline
\end{tabular}

Table 2. Description of white matter clusters showing lower fractional anisotropy (FA) in those with treatment-resistant schizophrenia (TRS) compared with either psychiatrically healthy controls or those with ultra-treatment-resistant schizophrenia (UTRS). Regions are shown for the Juelich histological white matter labels and tractography atlases and are ordered according to greatest probability of the cluster residing in each region (averaged across all voxels in the cluster). Any region with low $(<1 \%)$ probability of inclusion in a cluster has been excluded for ease of reporting only.

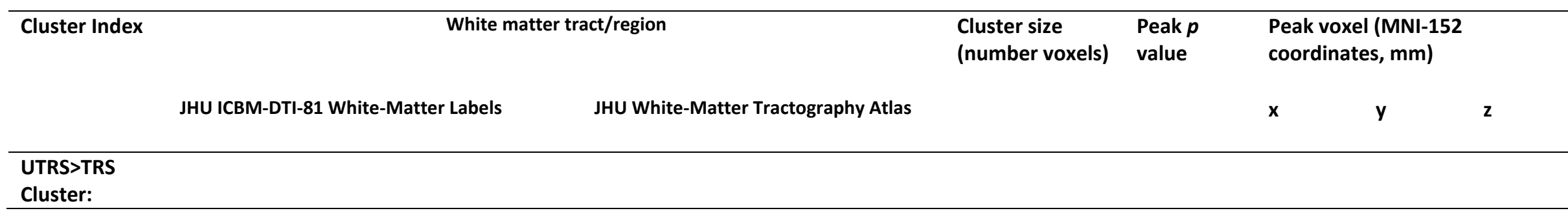


Superior longitudinal fasciculus ( $R$ )

\section{CON $>$ TRS}

\section{Cluster:}

7

Body of corpus callosum

Superior longitudinal fasciculus ( $R$ )

Splenium of corpus callosum

Superior corona radiata ( $R$ )

Posterior thalamic radiation (including optic

radiation, $\mathrm{R}$ )

Posterior corona radiata $(\mathrm{R})$

6

Posterior thalamic radiation (include optic radiation) (L)

Superior longitudinal fasciculus (L)

Sagittal stratum (include inferior longitidinal

fasciculus and inferior fronto-occipital

fasciculus, L)

5

Cerebral peduncle (bilateral)

Posterior limb of internal capsule (L)

Anterior limb of internal capsule (L)

Superior cerebellar peduncle (R)

Fornix (cres) / Stria terminalis (L)

Inferior cerebellar peduncle (R)

Superior cerebellar peduncle (L)

Superior longitudinal fasciculus (L)

Body of corpus callosum

Superior corona radiata $(\mathrm{L})$

Splenium of corpus callosum

Posterior corona radiata (L)

Anterior corona radiata (L)
Superior longitudinal fasciculus ( $R$ )

Inferior fronto-occipital fasciculus (R)

Forceps major

Inferior longitudinal fasciculus (R)

Corticospinal tract ( $R$ )

Superior longitudinal fasciculus

(temporal part, R)

Inferior longitudinal fasciculus (L)

Inferior fronto-occipital fasciculus (L)

Forceps major

Superior longitudinal fasciculus (L)

Superior longitudinal fasciculus

(temporal part, L)

Anterior thalamic radiation (L)

Corticospinal tract (bilateral)

Anterior thalamic radiation (R)

Superior longitudinal fasciculus (L)

Superior longitudinal fasciculus

(temporal part, L)

6150

4467
.022

74

92

100 
temporal part, R)

(temporal part, R) 5 